\title{
30
}

\section{Reliability Analysis of Load Path-Dependent Structures}

\author{
Wei Wang*, Ross B. Corotis ${ }^{\dagger}$, and Martin R. Ramirez $\ddagger$
}

*Research Associate, Wind Engineering Research Center, Texas Tech Univ., Lubbock, Texas 79409, USA

†Dean, College of Engineering and Applied Science, Univ. of Colorado, Boulder, Colorado 80309, USA

$\ddagger$ Dean, Greenfield Virtual Univ., UDM, 4001 West McNichols Rd., Detroit, Michigan 48219, USA

\section{INTRODUCTION}

The state of a structure can be described in terms of the complete set of state variables at any given time in its lifetime $[1,2,4,5,7]$. If the state of the structure is independent of the loading history, the structure is referred to as load pathindependent. For such a case, the state of the structure can be expressed in terms of the current values of the loads and common response statistics. In these cases, there exists a unique surface in the basic variable space which divides the space into two regions, representing safety and failure of the structure. This limit state can be written in the form $g(\mathbf{x})=0$. As will be discussed, the concept of such a function is not valid for load path-dependent structures. Instead, the concept of survival and failure paths must be used, and incipient failure directions determined for critical points.

As a first step toward the goal of developing general path-dependent methods which are more efficient than Monte Carlo methods and can provide insight into the nature of the problem, some fundamental concepts in time-varying reliability analysis of path-independent problems will be examined in the context of load path-dependency. New concepts that can help in the understanding of path-dependent reliability will be introduced.

\section{LIMIT STATE REGIONS IN THE BASIC VARIABLE SPACE}

Let $\mathbf{X}(t)$ be a vector of random processes, and define the basic variable space, $\mathcal{X}$, as a space whose axes are the sample space of each component of $\mathbf{X}(t)$. Then, for structures whose response is not load path-dependent, there is, in general, a unique limit state surface that divides $\mathcal{X}$ into a safe region and a failure region [10]. When the structural response is load path-dependent, however, there is no such division between a failure region and safe region $[8,12]$. At some point 
$\mathbf{x} \in \mathcal{X}$, the structure may or may not fail, depending on the path which the vector process $\mathbf{X}(t)$ has taken to reach $\mathbf{x}$. To see how the points in $\mathcal{X}$ are related to the state of the structure under investigation, the following definitions are helpful.

A structure is critical if an infinitesimal change of $\mathbf{X}(t)$ from its current value in the next moment will bring it to failure. A point $\mathbf{x} \in \mathcal{X}$ is a critical point if the structure with basic variables $\mathbf{X}(t)=\mathbf{x}$ is critical. A path to $\mathbf{X}$ is a sample function, $\mathbf{x}(\tau), \tau \leq t$, of the vector process $\mathbf{X}(t)$ with $\mathbf{x}(t)=\mathbf{x}$. A survival path to $\mathrm{x}$ is a path to $\mathbf{x}$, under which the structure has not failed before moment $t$, and is safe or critical at $t$. A final survival path to $\mathbf{x}$ is a path to $\mathbf{x}$ for which the structure may or may not have failed before moment $t$, but is safe or critical at $t$. A structure may have "failed" before moment $t$, but be safe at $t$ in the case, for example, of a serviceability limit state. A survival path to $\mathbf{x}$ is also a final survival path to $\mathbf{x}$. A final survival path may not be defined if some of the basic variable processes, such as material properties, have no definition after the failure of the structure. An immediate survival direction at $\mathbf{x}$ is a direction in which an infinitesimal step away from $\mathbf{x}$ will not cause failure to the structure. An immediate failure direction at $\mathbf{x}$ is a direction in which an infinitesimal step away from $\mathbf{x}$ will cause failure to the structure.

It appears that there are only three types of points in the basic variable space:

Type f: Points which have no survival path reaching them.

Type t: Points which for some survival paths reaching them, have both immediate survival and failure directions.

Type s: Points which have no immediate failure directions for any survival path that ends at them.

Regions in $\mathcal{X}$ can be defined correspondingly,

$\begin{array}{lll}\text { failure region: } & D_{f}=\{\text { All type f points } \\ \text { transition region: } & D_{t}=\text { A All type t points } \\ \text { safe region: } & D_{s}=\{\text { All type s points }\end{array}$

These three regions are mutually exclusive and collectively exhaustive.

For any point $\mathbf{x} \in D_{t}$, there will, in general, be paths to $\mathbf{x}$ which do not have failure directions (i.e., failure is not imminent). By the definition of a type $t$ point, however, there exists at least one path to $\mathbf{x}$ which has failure directions (i.e., $\mathbf{x}$ is critical). Define $q(\mathbf{x} ; t)$ to be the likelihood that $\mathbf{x}$ is critical. Then

$q(\mathbf{x} ; t)=q_{1}(\mathbf{x} ; t) q_{2}(\mathbf{x} ; t)$

where

$q_{1}(\mathbf{x} ; t) \equiv$ the likelihood that there is a survival path to $\mathbf{x}$

and

$\begin{aligned} q_{2}(\mathbf{x} ; t) \equiv & \text { the likelihood that there is an immediate failure } \\ & \text { direction at } \mathbf{x} \text { for any survival path to } \mathbf{x}\end{aligned}$

$q_{1}$ can be viewed as the traditional reliability function $L_{T}$ over space, and $q_{2}$ the traditional hazard function $h(t)$ over space. $q_{2}(\mathbf{x}, t)$ can be defined to equal unity over region $D_{f}$ without conflict with Eq. 4 . 
Regions $D_{s}, D_{t}$ and $\nu_{f}$, and quantities $q$ and $q_{2}$ are illustrated in Fig. 1 . It is conceivable that one or even two of the three regions may not exist, depending on the specific problem.

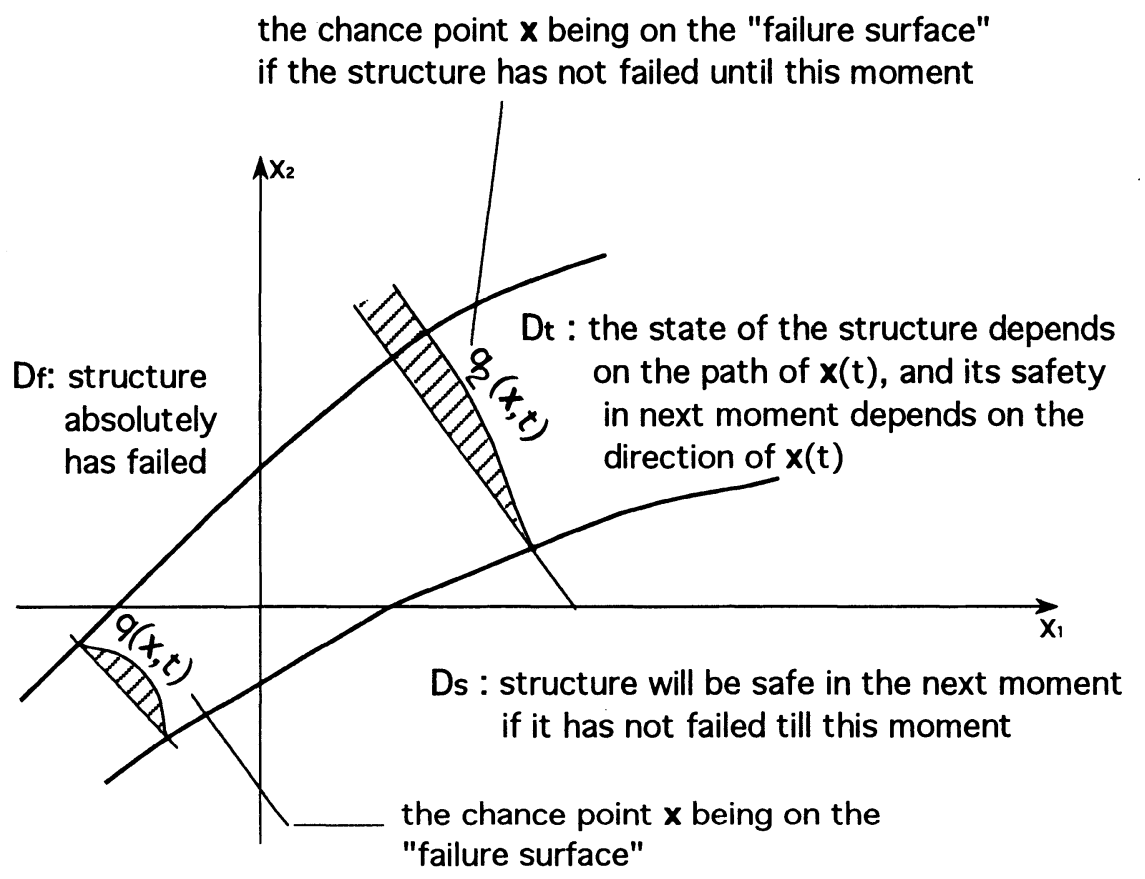

Fig. 1 Regions in the Basic Variable Space

Consider an arbitrary point $\mathbf{x} \in D_{t}$, and an arbitrary survival path to $\mathbf{x}$ which has failure directions at $\mathbf{x}$. If there exists a segment of surface which passes through $\mathbf{x}$ and separates the failure directions from the survival directions, this segment of surface can then be defined as the limit state surface of the structure at point $\mathbf{x}$ of path $\mathbf{x}(t)$. It is similar to the limit state surface in the path-independent problems, except that it must be defined pointwise and pathwise.

If the segment of the failure surface at $\mathbf{x}$ of path $\mathbf{x}(t)$ is smooth, it is necessarily a hyperplane. A failure direction, $\mathbf{n}$, of unit length perpendicular to the hyperplane can be defined as the unit outnormal vector to the failure surface at $\mathbf{x}$ by path $\mathbf{x}(t)$. It is dependent on path $\mathbf{x}(t)$ and time $t$ :

$\mathbf{N}=\mathbf{n}(\mathbf{X}(t) ; t)$

The upper case $\mathbf{N}$ is used in Eq. 5 to reflect the fact that the outnormal vector is a random quantity. The probability density function of $\mathbf{N}$ over the 
basic variable space $\mathcal{X}$, if it exists, should depend on time $\mathbf{t}$ and location $\mathbf{x}$, and therefore has the form,

$f_{\mathrm{N}}(\mathbf{n} ; \mathbf{x}, t)$

When the segment of surface is not smooth, a normal vector does not exist. The density function in Eq. 6 is nevertheless useful if it is redefined as the likelihood that an arbitrary direction $\mathbf{n}$ is an immediate failure direction.

With the basic definitions of regions and failure directions, it is possible to address the reliability problem in terms of vector outcrossing theory. A general approach is introduced next.

\section{OUTCROSSING RATE ANALYSIS}

In path-independent problems, the rate at which the basic variable processes cross the limit state surface into the failure zone can be related directly to the probability of failure of the structure if outcrossing is a rare event $[3,13]$. This should hold for path-dependent problems also, if the limit state surface is taken as defined in the previous section. The formulas for outcrossing rate computation are derived for path-dependent problems for the one- and multi- dimensional cases and for discrete $\mathbf{X}(t)$ in this section.

\section{1 upcrossing rate - one-dimensional case}

Consider a problem involving two random processes, the load process $X(t)$, and the barrier process $B(t)$. $B(t)$ depend on the entire history of $X$ up to time $t$. Failure is defined as $X \geq B$.

For an upcrossing to take place during $(t, t+\Delta t)$, the following conditions must be met [9]:

$X(t)<B(t)$ and $X(t+\Delta t)>B(t+\Delta t)$

That is, $X(t)$ must be below the barrier at time $t$ and above it at time $t+\Delta t$.

When $\Delta t$ is small, the approach of Rice [1944] can be used, and the second inequality in Eq. 7 can be written as

$X(t)+\dot{X}(t) \Delta t \geq B(t)+\dot{B}(t) \Delta t$

or equivalently,

$X(t) \geq B(t)-[\dot{X}(t)-\dot{B}(t)] \Delta t$

The conditions for an upcrossing to occur is thus,

$B(t) \geq X(t) \geq B(t)-[\dot{X}(t)-\dot{B}(t)] \Delta t$

Note that in Eq. $10, b(t)$ and $\dot{b}(t)$, realizations of $B(t)$ and $\dot{B}(t)$ respectively, are different for different sample functions, $x(\tau)$, of $X(\tau)(0 \leq \tau \leq t)$. This is the critical difference from the path-independent problems, where $b(t)$ and $\dot{b}(t)$ are constants for fixed $t, x$, and $\dot{x} . B(t)$ and $\dot{B}(t)$ now are also random variables whose distributions depend on the nature of the process $X(t)$. 
Assume that the joint distribution of $X(t), \dot{X}(t), B(t)$ and $\dot{B}(t)$ exists. The probability associated with Eq. 10 for given $b(t)$ and $\dot{b}(t)$ is the integration of the joint pdf over the shaded area in Fig. 2. Then the probability that an upcrossing (U.C.) takes place during $(t+\Delta t)$, is,

$P[$ U.C. $]=\int_{-\infty}^{\infty} d b \int_{-\infty}^{\infty} d \dot{b} \int_{\dot{b}(t)}^{\infty} d \dot{x} \int_{b(t)-[\dot{x}(t)-\dot{b}(t)] \Delta t}^{b(t)} f_{B(t), \dot{B}(t), X(t), \dot{X}(t)}(b, \dot{b}, x, \dot{x} ; t) d x(11)$

Equation 11 can be simplified using the mean value theorem for integration,

$P[U . C]=.\Delta t \int_{-\infty}^{\infty} d b \int_{-\infty}^{\infty} d \dot{b} \int_{\dot{b}(t)}^{\infty}[\dot{x}(t)-\dot{b}(t)] f_{B(t), \dot{B}(t), X(t), \dot{X}(t)}(b, \dot{b}, b, \dot{x} ; t) d \dot{x}$

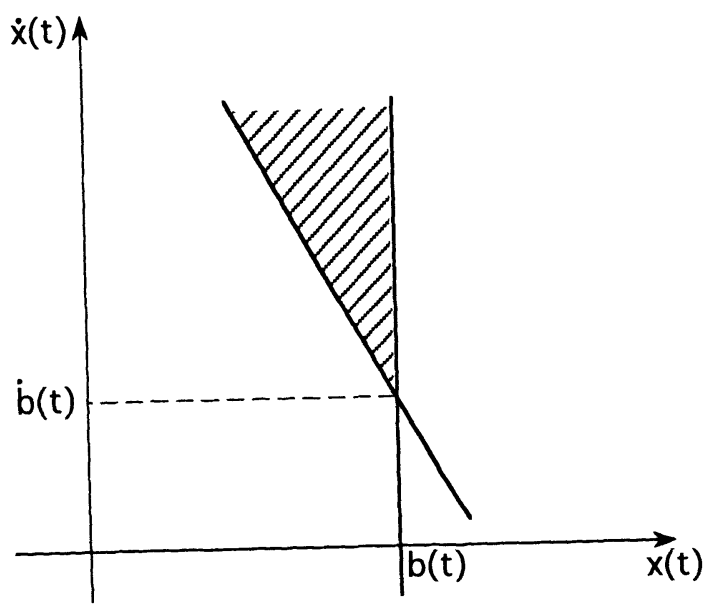

Fig. 2 Likelihood of an Upcrossing

Assume that $\Delta t$ is so small that the occurrence of more than one upcrossing during $(t, t+\Delta t)$ is negligible, and define $N^{+}$to be the number of upcrossings during this period. The expected number of outcrossings in $(t, t+\Delta t)$ is then,

$E\left[N^{+}\right]=1 \cdot \operatorname{Prob}[$ U.C. $]+0 \cdot \operatorname{Prob}[$ no U.C. $]=\operatorname{Prob}[U . C$.

Hence, the mean rate of upcrossing at time $t$ is given by,

$$
\begin{aligned}
\nu^{+}(t) & =\frac{E\left[N^{+}\right]}{\Delta t} \\
& =\int_{-\infty}^{\infty} d b \int_{-\infty}^{\infty} d \dot{b} \int_{\dot{b}(t)}^{\infty}[\dot{x}(t)-\dot{b}(t)] f_{B(t), \dot{B}(t), X(t), \dot{X}(t)}(b, \dot{b}, b, \dot{x} ; t) d \dot{x}
\end{aligned}
$$


or, equivalently,

$\nu^{+}(t)=\int_{-\infty}^{\infty} f_{X(t)}(b) d b \int_{-\infty}^{\infty} d \dot{b} \int_{\dot{b}(t)}^{\infty}[\dot{x}(t)-\dot{b}(t)] f_{(B(t), \dot{B}(t), \dot{X}(t)) \mid X(t)}(b, \dot{b}, \dot{x} ; t) d \dot{x}$

\subsection{Outcrossing Rate - Multi-Dimensional Case}

In outcrossing analysis, the attention is focused on the time period $(t, t+\Delta t)$ $[6,11]$. What happened before $t$ is not important as long as the structure is not in a failure state at $t$. Accordingly, for the convenience of outcrossing analysis, points in $\mathcal{X}$ can be re-classified using the concept of final survival path described previously.

Define,

Type fo: Points which have no final survival path entering them.

Type to: Points which for some final survival paths entering them, have both immediate survival and failure directions.

Type so: Points which have no failure directions for any final survival path that ends at them.

and, correspondingly,

$\begin{array}{lll}\text { failure region: } & D_{f o}=\{\text { All type fo points } \\ \text { transition region: } & D_{t o}=\text { A All type to points } \\ \text { safe region: } & D_{s o}=\{\text { All type so points }\end{array}$

$D_{s o}, D_{t o}$ and $D_{f o}$ are also mutually exclusive and collectively exhaustive.

With these definitions, outcrossings can only take place in region $D_{t o}$. A failure surface at point $\mathbf{x}$ for a path $\mathbf{x}(t)$ is now a segment of surface that divides the immediate failure directions from the immediate survival directions of a final survival path $\mathbf{x}(t)$. The chance that an arbitrary point $\mathbf{x} \in D_{t}$ is on a failure surface of a final survival path will be denoted as $q^{o}(\mathbf{x} ; t)$, which can be decomposed into two components,

$q^{o}(\mathbf{x} ; t)=q_{1}^{o}(\mathbf{x} ; t) q_{2}^{o}(\mathbf{x} ; t)$

where

$q_{1}^{o}(\mathbf{x} ; t) \equiv$ the likelihood that there is a final survival path to $\mathbf{x}$

and

$q_{2}^{o}(\mathbf{x} ; t) \equiv$ the likelihood that there is a failure direction at $\mathbf{x}$ for any final survival path to $\mathbf{x}$

Since a survival path is also a final survival path, the following relationships hold,

$D_{f} \supseteq D_{f o} \quad D_{t} \subseteq D_{t o} \quad D_{s} \supseteq D_{s o}$ 
Moreover,

$q_{1}^{o}(\mathbf{x} ; t) \geq q_{1}(\mathbf{x} ; t)$ and $q_{2}^{o}(\mathbf{x} ; t) \geq q_{2}(\mathbf{x} ; t)$

$\longrightarrow \quad q^{o}(\mathbf{x} ; t) \geq q(\mathbf{x} ; t)$

For any path reaching $\mathbf{x}$ at time $t$, only those velocity vectors, $\dot{\mathbf{X}}(t)$ that satisfy

$\dot{x}_{n} \equiv \dot{\mathbf{x}} \cdot \mathbf{n}>0$

will cause failure at the next moment, where $\mathbf{n}$ is the outnormal vector to a failure surface, or an immediate failure direction if the surface is not smooth.

Assume that the joint pdf of $\mathbf{N}(t), \mathbf{X}(t), \dot{\mathbf{X}}(t)$ at time $t$ exists. Emulating the extension of Rice's upcrossing formula to the multi-dimensional case [Melchers, 1987], the likelihood of an outcrossing at time $t$, which equals the mean rate of outcrossings at $t$ when outcrossing is a rare event, is

$\nu^{+}(t)=\int_{\mathcal{X}} d \mathbf{x} \iint_{\dot{x}_{n} \geq 0}(\dot{\mathbf{x}} \cdot \mathbf{n}) f_{(\mathbf{N}, \dot{\mathbf{x}}, \mathbf{X})}(\mathbf{n}, \dot{\mathbf{x}} ; \mathbf{x}, t) q^{o}(\mathbf{x} ; t) d \mathbf{n} d \dot{\mathbf{x}}$

In Eq. $24, q^{\circ}$ is the likelihood that $\mathbf{x}$ is on a limit state surface; $f_{\mathbf{N}, \dot{\mathbf{x}}, \mathbf{X}}$ is the likelihood that a path $\mathbf{x}(t)$ reaches point $\mathbf{x}$, having velocity vector $\dot{\mathbf{x}}$ and outnormal vector $\mathbf{n}$; and $\dot{\mathbf{x}} \cdot \mathbf{n}$ is analogous to $\dot{x}-\dot{b}$ in Eq. 15. $f_{\mathbf{N}, \dot{\mathbf{X}}, \mathbf{X}}$ vanishes in regions $D_{f o}$ and $D_{s o}$ by the definitions of these two regions.

Define,

$E^{+}\left[X_{n} \mid \mathbf{X}=\mathbf{x}\right]=\iint_{\dot{x}_{n}>0}(\dot{\mathbf{x}} \cdot \mathbf{n}) f_{(\mathbf{N}, \dot{\mathbf{X}}) \mid \mathbf{X}}(\mathbf{n}, \dot{\mathbf{x}} ; \mathbf{x}, t) q^{o}(\mathbf{x} ; t) d \mathbf{n} d \dot{\mathbf{x}}$

which determines the mean rate of outcrossing at a given point $\mathbf{x} \in \mathcal{X}$. Equation 25 then takes the form,

$\nu^{+}(t)=\int_{\mathcal{X}} E^{+}\left[X_{n} \mid \mathbf{X}=\mathbf{x}\right] f_{\mathbf{X}}(\mathbf{x} ; t) d \mathbf{x}$

where $f_{\mathbf{X}}$ is the joint pdf of $\mathbf{X}$. Equation 26 is very similar to its counterpart for the path-independent problems. However, the integrand and the integration domain here have quite different interpretations.

\section{SUMMARY AND CONCLUSION}

The concept of limit state surface in the basic variable space is discussed in this paper. The traditional concept of limit state surface for the load pathindependent problems is not applicable to load path-dependent problems. Instead, there is a transition zone in which each point may be on a limit state surface, which is a segment of surface that separates the immediate failure directions from the immediate survival directions. This piece of failure surface is in general different for different points in the transition zone, and is different for different paths to the same point.

Formulas computing the outcrossing rates are derived for load path-dependent problems. It can be observed that the major difference between these formulas and their counterparts in the path-independent problems is that an extra term, which represents the chance that the current point is on a limit state surface, is present in the path-dependent formulation. 


\section{ACKNOWLEDGMENT}

The support of the Department of Civil Engineering, The Johns Hopkins University, and the National Science Foundation, grant MSS-9016018, is gratefully acknowledged.

\section{REFERENCES}

[1] Bogdanoff, J.L., Kozin, F. (1985). Probabilistic Models of Cumulative Damage. John Wiley \& Sons, New York.

[2] Cordts, D., Kollmann, F. G. (1986). "An Implicit Time Integration Scheme for Inelastic Constitutive Equations With Internal State Variables." Int $J$ for Num Meth in Eng, v 23 p 533-554.

[3] Ditlevsen, O. (1983). "Gaussian Outcrossings From Safe Convex Polyhedrons". J of Eng Mech Div, ASCE, v 109, n 1, p 127-148.

[4] Ditlevsen, O. (1990). " Asymptotic First-Passage Time Distributions in Compound Poisson Processes." Structural Safety, v 8, p 327-336.

[5] Ditlevsen, O. (1991). " Gaussian Excited Elasto-Plastic Oscillator With Rare Visits to the Plastic Domain." J of Sound \& Vib, v 145, n 3, p 443-456.

[6] Hagen, O. and Tvedt, L. (1991). "Vector Process Out- Crossing as Parallel System Sensitivity Measure." J Eng Mech, ASCE, v 117, n 10, p 2201-2220.

[7] Mukherjee, S. (1982). Boundary Element Methods in Creep and Fracture. Applied Science Publishers, New York.

[8] Rackwitz, R., and Fiessler, B. (1978). "Structural Reliability Under Combined Random Load Sequences." Computers and Structures, v 9, n 2 p 489494 .

[9] Rice, S. O. (1944). "Mathematical Analysis of Random Noise." Bell System Technical Journal, v 23, n 282.

[10] Thoft-Christensen, P., Murotsu, Y. (1986). Application of Structural Systems Reliability Theory. Springer-Verlag, New York.

[11] Veneziano, D., Grigoriu, M., Cornell, C. A. (1977). "Vector-Process Models for System Reliability." J Engng Mech Div, ASCE, v 103, n EM3, p 441-460.

[12] Wang, W., Corotis, R. B., Ramirez, M. R. (1993). "Modal Failure Probability and System Performance." Structural Engineering in Natural Hazards Mitigation, Proc Structural Congress'93. v 2, p 1312-1317.

[13] Wen, Y. K. and Chen, H.-C. (1989). "System Reliability Under Time Varying Loads: I." J Eng Mech, ASCE, v 115, n 4, p 808-823. 